\title{
PRIMER REGISTRO DE THALASSIONEMA PSEUDONITZSCHIOIDES (SCHUETTE \& SCHRADER) HASLE (THALASSIONEMATACEAE, BACILLARIOPHYCEAE) EN EL PACIFICO SURORIENTAL FRENTE A LA COSTA DE CHILE
}

\author{
FIRST RECORD OF THALASSIONEMA PSEUDONITZSCHIOIDES \\ (SCHUETTE \& SCHRADER) HASLE (THALASSIONEMATACEAE, \\ BACILLARIOPHYCEAE) IN THE SOUTHEASTERN PACIFIC OFF THE \\ COAST OF CHILE
}

\author{
Patricio Rivera ${ }^{1}$, Fabiola Cruces $^{1} \&$ Sergio Avaria ${ }^{2}$ \\ 'Departamento de Botánica, Facultad de Ciencias Naturales y Oceanográficas, Universidad de Concepción, \\ Casilla 160-C, Concepción, Chile. E-mail: privera@udec.cl \\ ${ }^{2}$ Facultad de Ciencias del Mar, Universidad de Valparaíso, Casilla 5080, Reñaca, Chile.
}

\begin{abstract}
This note reports the finding of Thalassionema pseudonitzschioides (Schuette \& Schrader) Hasle in samples collected in Iquique Bay $\left(20^{\circ} 12^{\prime} \mathrm{S}, 70^{\circ} 10^{\prime} \mathrm{W}\right)$, in northern Chile. This is the first record of the species for the region. The taxon has been associated mainly with subtropical to temperate waters.
\end{abstract}

Las especies del género Thalassionema se distribuyen fundamentalmente en aguas marinas subtropicales y temperadas, aunque $T$. nitzschioides es un taxón cosmopolita. Thalassionema nitzschioides (Grunow) Mereschkowsky, T. frauenfeldii (Grunow) Hallegraeff y $T$. bacillare (Heiden) Kolbe son las únicas especies del género que han sido señaladas hasta la fecha para las aguas marinas de Chile (Rivera 1983; Rivera et al. 1990; Koizumi 1990; Vera et al. 1996; Avaria et al. 1999; Romero \& Hebbeln 2003; Avaria et al. 2003; Avaria et al. 2004). Las dos primeras están presentes en gran parte del litoral y T. nitzschioides es particularmente abundante al sur de los 33 grados de latitud sur.

La presente nota informa el hallazgo de otra especie de este género, T. pseudonitzschioides (Schuette \& Schrader) Hasle in Hasle \& Syvertsen (1996), en muestras recolectadas en la Bahía de Iquique, situada en la zona norte de Chile $\left(20^{\circ} 12^{\prime} \mathrm{S}, 70^{\circ} 10^{\prime} \mathrm{W}\right)$. Este material se encuentra depositado en la colección Diatomológica de la Universidad de Concepción (DIAT-CONC). Más de un centenar de ejemplares fueron estudiados. Las células de $T$. pseudonitzschioides se unen por sus extremos formando cadenas estrelladas y en zig-zag (Fig. 1). Fotografías obtenidas con el microscopio electrónico de barrido nos permitieron observar los caracteres distintivos de esta especie. Las valvas son heteropolares (Figs. 2, 4), 34-45 $\mu \mathrm{m}$ de largo y 3,5-4,5 $\mu \mathrm{m}$ de ancho, provistas en el lado externo de uno de los extremos de una prolongación con forma de punta de flecha (Fig. 3), las aberturas externas de las aréolas (9-11 en $10 \mu \mathrm{m})$ están cruzadas por una barra en forma de "Y" (Fig. 7), y en un extremo valvar los labios del proceso labiado se orientan en forma oblicua al eje apical de la célula (Fig. 5), pero son casi perpendiculares a él en el proceso labiado del extremo opuesto (Fig. 6). 

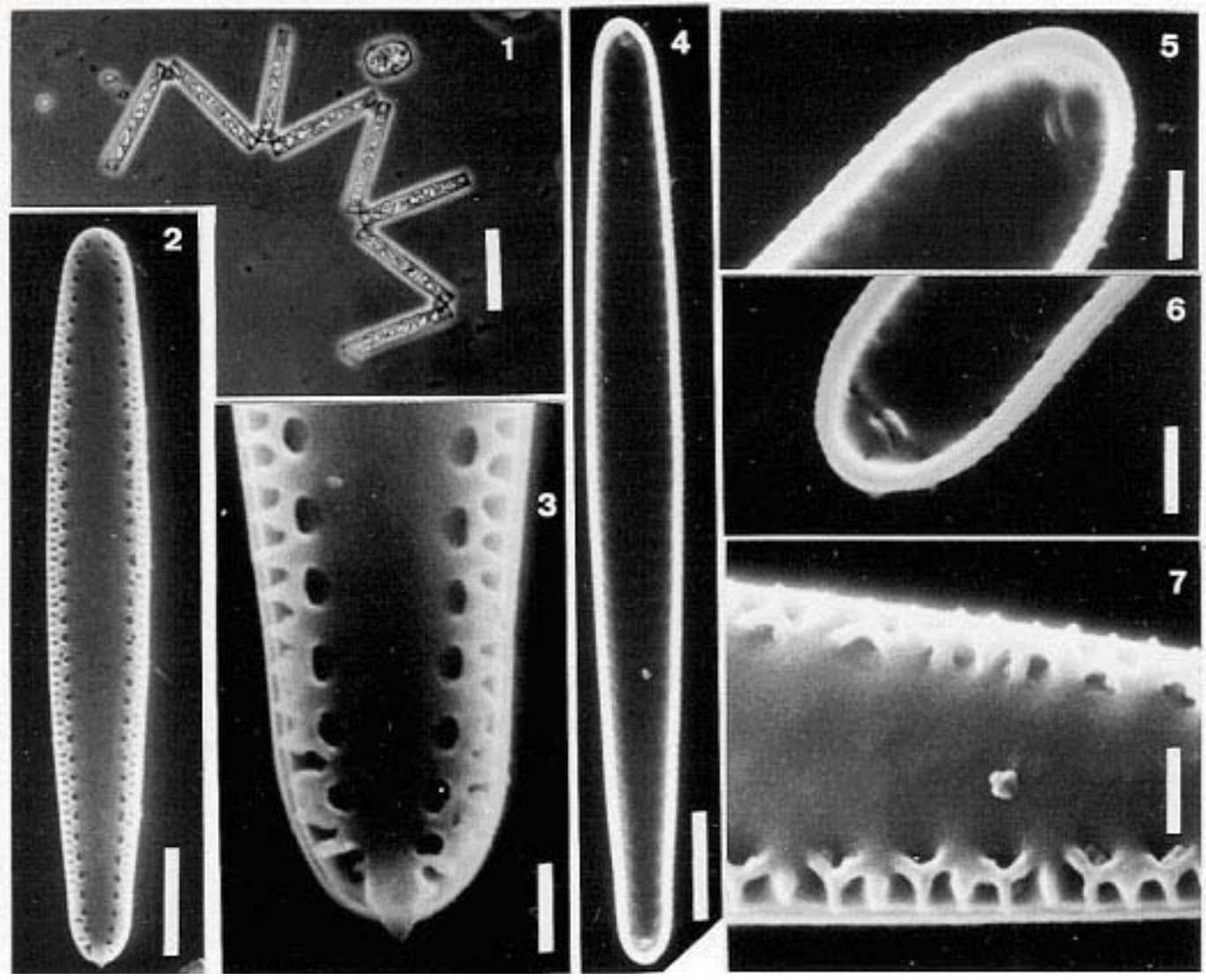

Figuras 1-7. Thalassionema pseudonitzschioides (Schuette \& Schrader) Hasle. Fig. 1, ML; Figs. 2-7, MEB. Escala: 30 $\mu \mathrm{m}$ (Fig. 1), $5 \mu \mathrm{m}$ (Figs. 2, 4), $1 \mu \mathrm{m}$ (Figs. 3-7). Fig. 1. Células unidas por mucopolisacárido, formándose una cadena estrellada y en zig-zag. Fig. 2. Valva heteropolar, vista exterior. Fig. 3. Extremo de la valva provisto de una prolongación en forma de punta de flecha. Fig. 4. Valva heteropolar, vista interior. Un proceso labiado en cada extremo. Fig. 5. En un extremo valvar, el proceso labiado se orienta en forma oblicua al eje apical de la célula. Fig. 6 . En el otro extremo valvar, el proceso labiado se orienta en forma casi perpendicular al eje apical. Fig. 7. Las aberturas externas de las aréolas están cruzadas por una barra en forma de "Y". (ML: microscopía fotónica. MEB: microscopía electrónica de barrido).

La muestra recolectada en abril de 1982 estuvo dominada por Thalassiosira subtilis (Ostenfeld) Gran emend. Hasle y en menor grado por diversas especies del género Chaetoceros Ehrenberg. En las dos muestras obtenidas en noviembre de 2003, además de Thalassiosira angustelineata (A. Schmidt) Fryxell \& Hasle y de Odontella longicruris (Greville) Hoban, fue notoriamente abundante Thalassiosira minuscula Krasske, acompañada por algunas especies de Chaetoceros.

Hallegraeff (1986) coloca a Thalassionema pseudonitzschioides como sinónimo de $T$. nitzschioides, y Moreno-Ruiz \& Licea (1995) como sinónimo de $T$. nitzschioides var. claviforme
(Schrader) Moreno-Ruiz. Sin embargo, en estudios más recientes como el de Hasle \& Syvertsen (1996), y muy especialmente en la revisión de la Familia Thalassionemataceae realizada por Hasle (2001), se trata a T. pseudonitzschioides como una especie independiente. Compartimos esta decisión sobre la base que en T. nitzschioides los extremos valvares presentan la misma forma, las aberturas externas de las areolas poseen otro tipo de oclusión y los procesos labiados se orientan paralelos al eje apical de la célula.

T. pseudonitzschioides es considerado por Hasle (2001) como un taxón propio de aguas subtropicales a temperadas. Descrito originalmente 
para el Golfo de California (Schuette \& Schrader 1982, como Thalassiothrix pseudonitzschioides), también se ha señalado para el Océano Indico Tropical (Jousé et al. 1977). Hasle (2001) lo señala para algunas localidades en el Atlántico Norte, Atlántico Sur, Pacífico Norte y para Ecuador y Melbourne, Australia, en el Pacífico Sur.

MATERIAL DE ESTUDIO: DIAT-CONC M-1561, Iquique, Erfen IV/202, abril 1982; DIAT-CONC M3225 , Bahía de Iquique, $20^{\circ} 12^{\prime} \mathrm{S}, 70^{\circ} 10^{\prime} \mathrm{W}$, 10.11.2003; DIAT-CONC M-3226, Bahía de Iquique, $20^{\circ} 12^{\prime} \mathrm{S}, 70^{\circ} 10^{\prime} \mathrm{W}, 11.11 .2003$.

\section{AGRADECIMIENTOS}

Agradecemos las sugerencias y comentarios efectuados al manuscrito por el Dr. C. Lusk y por dos revisores anónimos. También se agradece la colaboración del personal del Laboratorio de Microscopía Electrónica de la Universidad de Concepción, en particular de los señores Raúl Alarcón, Hugo Pacheco y Julio Pugin.

\section{BIBLIOGRAFIA}

Avaria, S., L. Jorquera, P. Muñoz \& P. Vera. 1999. Distribución del microfitoplancton marino en la zona de aguas interiores comprendida entre el Golfo de Penas y el Estrecho de Magallanes, Chile, en la primavera de 1996 (Crucero Cimar-Fiordo II). Ciencia y Tecnología del Mar 22: 81-110.

Avaria, S., C. CÁceres, P. CAstillo \& P. Muñoz. 2003. Distribución del microfitoplancton marino en la zona Estrecho de Magallanes-Cabo de Hornos, Chile, en la primavera de 1998 (Crucero Cimar 3 Fiordos). Ciencia y Tecnología del Mar 26: 79-96.

Avaria, S., C. CAceres \& P. MuÑoz. 2004. Distribución del microfitoplancton marino entre el Golfo Corcovado y el Estero Elefantes en la primavera de 1998 y en el verano de 1999 (Crucero Cimar 4 Fiordos). Ciencia y Tecnología del Mar 27: 17-47.

HallegraefF, G.M. 1986. Taxonomy and morphology of the marine plankton diatoms Thalassionema and Thalassiothrix. Diatom Research 1: 57-80.

Hasle, G.R. 2001. The marine, planktonic diatom Family Thalassionemataceae: morphology, taxonomy and distribution. Diatom Research 16: 1-82.

Hasle, G.R. \& E.E. Syvertsen. 1996. Marine Diatoms. En: Identifying Marine Diatoms and Dinoflagellates (ed. C.R. Tomas,), pp. 5-385. Academic Press, San Diego.

Jouse, A.P., V.V. MuKhina, G.K. Kazarina \& O.G. Koslova. 1977. Atlas of Microorganisms in Bottom Sediments of the Oceans. Nauka, Moscow. 33 pp., 160 pls.

KoIzUMI, I. 1990. Miocene to Pliocene marine Diatoms from Caleta Herradura de Mejillones Section, Chile. Reports of Andean Studies, Shizuoka University 3: 17-32.

Moreno-Ruiz, J.L. \& S. Licea. 1995. Observations on the valve morphology of Thalassionema nitzschioides (Grunow) Hustedt. En: Proceedings of the Thirteenth International Diatom Symposium (eds. D. Marino \& M. Montresor), pp. 393-413. Biopress Limited, Bristol, England.

Rivera, P. 1983. A Guide for References and Distribution for the Class Bacillariophyceae in Chile between $18^{\circ} 28^{\prime} \mathrm{S}$ and $58^{\circ} \mathrm{S}$. Bibliotheca Diatomologica, J. Cramer, Vaduz, Liechtenstein. Band 3, 386 pp.

Rivera, P., M. Gebauer \& H.L. Barrales. 1990. A Guide for References and Distribution for the Class Bacillariophyceae in Chile between $18^{\circ} 28^{\prime} \mathrm{S}$ and $58^{\circ}$ S. Part II. Data from 1982 to 1988 . Gayana Botanica 46: 155-198.

Romero, O. \& D. Hebbeln. 2003. Biogenic silica and diatom thanatocoenosis in surface sediments below the Peru-Chile Current: controlling mechanisms and relationship with productivity of surface waters. Marine Micropaleontology 48: 71-90.

Schuette, G. \& H. Schrader. 1982. Thalassiothrix pseudonitzschioides sp. nov.; a common pennate diatom from the Gulf of California. Bacillaria 5: 213-223.

Vera, P., S. Avaria \& P. Muñoz. 1996. Composición y distribución del fitoplancton de los fiordos adyacentes a Campos de Hielo Sur y su relación con algunos parámetros oceanográficos. Ciencia y Tecnología del Mar 19: 73-92.

Fecha de recepción: 29.09.04

Fecha de aceptación: 28.10.04 